regression separately for 1996-2003 and 2004-2013 to account for non-linear trends. Regional person-linked data from the Oxford Region and WA were used to account for the effect of transfers and coronary procedure admissions on trends.

Results From 1996-2013, there were 4.9 million CHD hospitalisations in England and 2.6 million in Australia (67\% men). From 1996-2003, there was between-country variation in the direction of trends in acute coronary syndromes (ACS) and chronic CHD hospitalisation rates $(\mathrm{p}<0.001)$. During 20042013, reductions in ACS hospitalisation rates were greater than for chronic CHD hospitalisation rates in both countries, with the largest subgroup declines in unstable angina [England: men $-7.1 \% /$ year (95\% CI -7.2 to -7.0 ), women $-7.5 \%$ / year $(-7.7$ to -7.3$)$; Australia: men $-8.5 \% /$ year (-8.6 to -8.4$)$, women $-8.6 \% /$ year $(-8.8$ to -8.4$)$ ]. Age-specific trends generally reflected overall downward trends in each subgroup except for MI rates in women aged 35-54 years in 2004-2013, [England: $0 \% /$ year $(-0.5$ to +0.4$)$; Australia: $+1.9 \% /$ year $(+1.4$ to +2.4$)]$. Rates of 'Other CHD' increased in 75-84 year olds in both countries. Chronic CHD comprised half of all CHD admissions, with the majority involving angiography or revascularisation. Analysis of linked regional data found increasing MI rates in WA from 2004-2013 for men and women. In both regions, an increasing proportion of admissions for other CHD were for coronary procedures (mainly angiogram) in Oxford (71\% in $1996 ; 84 \%$ in 2013) and WA (88\% in 1996 ; $91 \%$ in 2013).

Conclusion Since 2004, rates of all CHD subgroups have fallen in both countries, with greater declines in acute than chronic presentations. The slower declines and high proportion of chronic CHD admissions involving coronary procedures requires greater focus. Differing MI trends in younger women in both countries warrant further investigation.

\section{P61 INCIDENCE, RISK FACTORS AND PROGNOSE OF ACUTE KIDNEY INJURY IN CRITICALLY ILL PATIENTS: A PROSPECTIVE COHORT STUDY IN BRAZILIAN AMAZON}

${ }^{1}$ DMT Zanetta*, ${ }^{2}$ EA Burdmann, ${ }^{3}$ FAF Melo. ${ }^{1}$ School of Public Health, University of Sao Paulo, Sao Paulo, Brazil; ${ }^{2}$ Medical School, University of Sao Paulo, Sao Paulo, Brazil; ${ }^{3}$ Medical School, Acre Federal University, Rio Branco, Brazil

10.1136/jech-2019-SSMabstracts.212

Background Epidemiological studies, mainly prospective population-based studies, of acute kidney injury (AKI) are still scant, especially in low-income and middle-income countries ( $>85 \%$ of the world's population). This study aimed to identify incidence and factors associated AKI in critically ill patients of Brazilian Amazon, a region with limited health care facilities.

Methods Prospective cohort study of all adult patients without chronic renal disease admitted, and staying $>2$ days, in all Intensive Care Units(ICU) of Rio Branco, the largest city in Acre State, covering approximately $70 \%$ of the state population (800,000 inhabitants), from Feb 2014 to Feb 2016. Incidence, risk factors and outcomes of AKI (diagnosed by KDIGO criteria) were evaluated. Patients were followed for up to 7 days, discharge or death. Mortality was assessed $30 \mathrm{~d}$ after ICU discharge. Factors associated with AKI development and, in those with incident AKI, associated with mortality were evaluated in multiple logistic regression analysis. The proportional multiple Cox analysis evaluated 30-day mortality in non-AKI and AKI patients, using SPSS(v.22.0) software.

Results Of 1,494 patients admitted, 1,073 fulfilled selection criteria. AKI incidence was 52\% (Stage $1=62.1 \%, 2=15.6 \%$ and $3=22.2 \% ; 8.2 \%$ received dialysis). $60 \%$ of patients were admitted due to clinical condition, 25\% were hemodynamically unstable, $19 \%$ had respiratory failure. Only $2.2 \%$ had tropical diseases. Risk factors for AKI were age (adjusted OR (aOR) 1.2[CI95\% 1.1-1.3 for 10 years increase]), positive fluid balance $>1500 \mathrm{ml} / 24 \mathrm{~h}$ (aOR 2.9[2.1-3.9]), APACHE score (aOR 1.06[1.04-1.07 per unit increase]), clinical patients (reference surgical: aOR 1.6[1.2-2.6]). AKI had higher ICU mortality (AKI 43.4\% vs non-AKI 13.9\%). AKI mortality was associated with age (aOR 1.3[1.1-1.4 for 10 years increase]), mechanical ventilation (aOR 5.2[3.0-9.0]), KDIGO stage 3 (ref 1) (aOR 1.6[1.03-2.5]), vasoactive drugs or shock (aOR 2.6[1.4-4.7]), and sepsis (aOR 2.3[1.6-4.7]). Adjusted AKI hazard for 30 days after ICU discharge mortality was 1.8 (1.1-3.0).

Conclusion AKI incidence was strikingly high in critically ill patients in the Brazilian Amazon. Hospitalizations due to tropical diseases were rare, likely due to particular conditions of the Amazon area, with difficulty access to larger cities and limited health care facilities. AKI etiology and risk factors were similar to those seen in developed countries. However, mortality rates were higher. The follow-up of the cohort was for7 days and data collected may not represent all factors affecting outcomes. Poor social-economic conditions and infrastructure of health services may explain the high incidence and mortality rates for AKI observed. The results may contribute to the care of this group of patients.

\section{P62 HOW MUCH OF THE DISABILITY-RELATED INEQUALITIES IN HEALTH AND WELL-BEING ARE MEDIATED BY BARRIERS TO PARTICIPATION FACED BY PEOPLE WITH DISABILITIES? A CAUSAL MEDIATION ANALYSIS USING LONGITUDINAL DATA FROM WORKING AGE PEOPLE WITH AND WITHOUT DISABILITIES IN GREAT BRITAIN}

${ }^{1} Z$ Aitken*, 'G Disney, 'L Krnjacki, 'A Milner, ${ }^{2} E$ Emerson, ${ }^{1}$ AM Kavanagh. 'Melbourne School of Population and Global Health, The University of Melbourne, Melbourne, Australia; ${ }^{2}$ Centre for Disability Research, Faculty of Health and Medicine, Lancaster University, Lancaster, UK

\subsection{6/jech-2019-SSMabstracts.213}

Background Large health inequalities exist between people with and without disabilities, including many health conditions unrelated to the impairment causing the disability. There is some evidence that these health inequalities are, in part, due to the poor socio-economic circumstances experienced by people with disabilities, and therefore are amenable to public health intervention. In this study, we used a unique dataset to examine the relationship between disability acquisition and subsequent health outcomes using five different measures of health and wellbeing. We quantified the extent to which social barriers to participation explained the health inequalities between people with and without disability.

Methods We used data from three waves of the Life Opportunities Survey, a longitudinal study of disability in Great Britain with a strong focus on barriers to participation in society. We compared health and well-being outcomes between adults who recently acquired an impairment and those who remained disability-free, adjusting for baseline demographic, socio-economic 